UCRL- 91940

PREPRINT

\title{
RECENT PROGRESS OF THE ADVANCED TEST ACCELERATOR
}

\author{
D. S. Prono
}

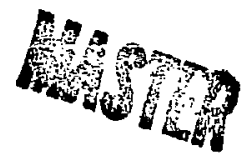

This paper was prepared for submittal to 1985 Particle Accelerator Conference Vancouver, B.C., Canada

May 13-16, 1985

Niay 13,1985

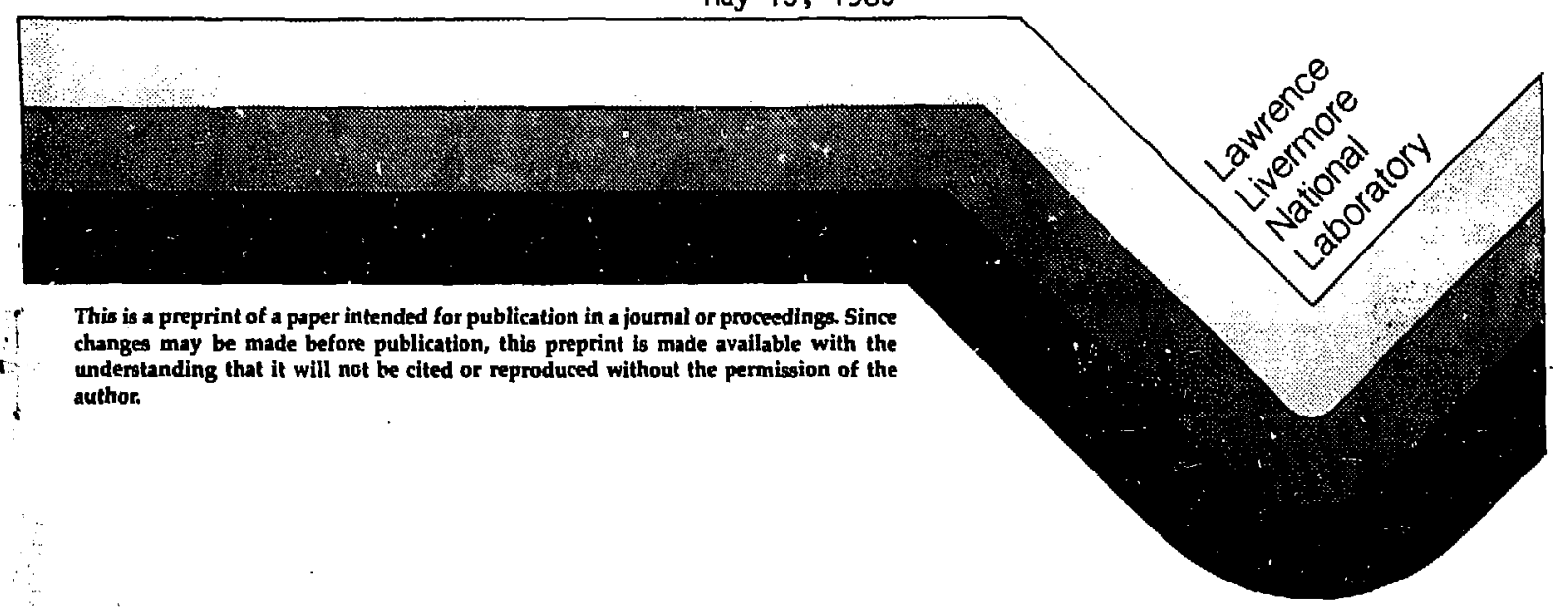




\section{RECENT PROGRESS OF THE ADVANCED TEST ACCELERATOR*}

D. S. Prono and the Beam Research Group

Lawrence Livermore National Laboratory University of California

Livermore, California

UCRL--91940

DE86 001579

May 13,1985

INTRODUCTION: The Challenge of High Current Transport Through an Accelerator

The Advanced Test Accelerator (ATA) of Lawrence Livermore National

Laboratory is a linear induction accelerator whose electron bean parameters are $10 \mathrm{kA}, 50 \mathrm{MeV}$, and $70 \mathrm{~ns}$. This accelerator structure basically is a $2.5 \mathrm{MeV}$ injector followed by 190 fdentical induction accelerator cores each of which incrementally adds $250 \mathrm{kV}$ to the electron beam as it threads the center of the core. Shown in Fig. 1 is one induction accelerator core; the primary components include the input power feed from the blumleins, the ofl to vacuum insulator, the ferrite which increases the inductance of the shorted turn input power feed, the ferrite reflector which damp modes in the vacuum cavity, and finaliy the acceleration gap across which the inductive accelerating electric field appears. With this surprisingly simple structure, high current electron beams can efficiently be accelerated; however, beam transport through a linear induction accelerator where there are many such accelerating cores is a very formidable challenge, primarily because of the difficulties presented

*Work performed jointly under the auspices of the U. S. Department of Energy by LLNL under contract $W-7405-E N G-4 B$ and for the Department of Defense under DARPA ARPA Order No. 4395 Amendment No. 31, monitored by NSWC under document number N60927-85-POWODO1; and SDIO/BMD-ATC MIPR No. W3-RPD-53-A127. 
by the discontinuity of the accelerating gap itself. In a smooth pipe, the self-radial electric and azimuthal magnetic field of the electron beam are terminated by the pipe wa11, a condtion invalidated under the accelerating gap. Thus, an electron beam ever so slightly off axis while under the accelerating gais has its dipole magnetic fields excite the accelerating gap and cavity. This results in non-symetric cavity modes feeding back onto the beam and inparting a slight transverse impulse. Between adjacent accelerator cores, this transverse impulse grows to a slight transverse displacement which then more strongly excites the next cavity. The very rapid growth of the beam's transverse displacement makes this instability, termed Beam Break-Up (BBU), the most virulent of instabilities plaguing induction accelerators. In this asymptotic limit, it is predicted that an initial small displacement, $\xi_{0}$. will grow to a value $\xi$ after passage through $N_{c}$ accelerator cores, where $\xi$ is given by

$$
\xi=\xi_{0} \exp \left[K \frac{\omega I_{B}^{N}\left(z_{1} / Q\right) Q}{B_{z}}\right] .
$$

Here, $\omega$ is the frequency of the most strongly excited asymetric cavity mode, $I_{b}$ is the electron beam current, $z_{\perp} / Q$ is the transverse shunt impedance, $Q$ is the typical cavity parameter, $B_{z}$ is the axial magnetic field used to focus the beam and $K$ reflects gap physical parameters. For ATA, $K=1.2 \times 10^{-13}$, $N_{c}=190, W=800 \mathrm{MHz}, Q=4, Z_{1} / Q=6.9, I_{b}=10 \mathrm{kA}$, and $B_{z}=3 \mathrm{~kg}$ which results in approximately 11 e-folds of growth. Figures $2 a$ and $2 b$ show how this severe growth manifests itself in degraded beam performance. Along the length of ATA are small magnetic induction loops which sense the time changing $\dot{B}_{\theta}$ fleld. In Fig. 2a, we show the signal output from magnetic induction loops 
as one progresses down the length of the accelerator. The loop located nearest the injector shows the inductive field associated with only the rise and fall of the electron beam pulse. As one progresses down the accelerator, this beginning and end of the induction signal becomes distorted due to difficulties in smoothly transporting a time varying current, but of more interest is the rapid growth of the $800 \mathrm{MHz}$ oscillations characterizing B8U growth of transverse beam motion. So severe is this growth that the beam actually is intercepting the side walls of the accelerator $(7 \mathrm{~cm}$ radius) causing disruption of the current pulse. This disruption is shown in Fig. 2b where beam current monitors alonis the accelerator length show an increasing truncation of the transported current pulse duration. Clearly, a means to suppress this instability is required to enable the beam to exit the accelerator with a full current and pulse duration. But also the instability should be early stabilized to minimize any growth of transverse motion which, for most applications of high-intensity electron beams, mast be damped out thereby increasing beam emittance and equilibrium beam size.

THE SOLUTION: The Concept and Practical Reality

What is required is a continuous, very strong focusing system which not only tightly constrains the electron beam to the accelerator centerline, but also phase mix damps any coherent transverse beam momentum into an overall increase in beam emittance. Higher order magnetic systems can accomplish these ends but are limited in versatility and difficult, if not expensive, to get strong field gradients near the accelerator centerline. An alternative choice is a continuous electrostatic system whereby one establishes a positive line of charge the entire length of the accelerator and to inject the electron 
beam onto this axis. The positive line charge has a symetric radial electric field that attracts and strongly focuses the electron beam onto the centerline axis. In addition, the radial electric field of the line charge forms an anharmonic potential well within which the restoring force is inversely proportional to the radial distance from the centerline axis. Thus, the beam electrons confined within the anharmonic potential well oscillate at different frequencies depending on their radial displacement. This spread in oscillating frequencies will cause electrons that initially form a displaced portion of the beam to be spatially randomized during transport in the anharmonic potential well, i.e., orbital phase mixing. Previous experiments at LLNL using the ETA, demonstrated the effectiveness of a short $(1.5 \mathrm{~m})$ positive line of charge in stabilizing transverse beam motion. ${ }^{2}$ In these highty successful experiments, the positive line charge was created by using the high-intensity electron beam to induce positive charges on a resistive graphite thread that was supported along the axis of the accelerator. This technique, effective though it is, is not feasible for creating a positive line charge the full length of a $100 \mathrm{~m}$ accelerator like the ATA.

Instead, the following technique, laser-guiding, is used. A vacuum base pressure of $10^{-4} \mathrm{~Pa}$ is achleved throughout the length of the accelerator. Benzene gas $\left(\mathrm{C}_{6} \mathrm{H}_{6}\right)$ is then carefully bled into the accelerator in such a way that an exially uniform elevated pressure of $10^{-2} \mathrm{~Pa}$ is created. Since the injector (the electron beam source) of the ATA is co-linear with the entire accelerator structure, we can direct a low-energy, small-diameter KrF laser beam through the injector and down the length of the accelerator. (The KrF laser parameters were $0.4 \mathrm{~J}$ energy, $28 \mathrm{~ns}$ pulse length, and 50 prad divergence.) 
We selected the $249 \mathrm{~nm}$ wavelength $\mathrm{KrF}$ laser and the benzene gas on the basis of a survey of phr,to-ionization cross sections which indicated that, through a twophoton ionization process, the fraction of ionized benzene could approach $20 \%$.

The laser is fired about $100 \mathrm{~ns}$ before the injector launches the electron beam into the accelerator. The laser pulse partlally lonizes the benzene and forms a continuous, narrow, straight plasma channel of benzene ions and free electrons. The low energy of the KrF laser used in the ATA limits the lonization fraction of the benzene to $1 \%$. In the brief $100 \mathrm{~ns}$ interval after passage of the laser pulse but vefore injection of the electrons, the plasma, with its heavy benzene ions, experiences almost no recombination or spatial diffusion.

To form the positive line charge (that is, a column containing enly benzene ions), the high-intensity electron beam is infected onto the preformed plasma column (see fig. 3). The free benzene electrons are ejected by the strong radial electric field at the head of the electron beam. To avoid accumulation of these free electrons and to ensure that their ejection from the ion channel is complete, the axlal magnetic fleld for the entire length of the accelerator is turned off. Once the secondary electrons are ejected, the dominant force acting on the beam electrons is the focusing electric field of the ion column since the electron beams self fields cancel to order $1 / \gamma^{2}$.

\section{RESULTS: Laser Guiding is a Technological Breakthrough}

The impact of laser-guiding transport through the accelerator is shown in Fig. 4, which is to be compared to the data shown in Figs. $2 a$ and $2 b$. We see that there is virtually no apparent growth of the BBU instability and full beam current and pulse duration is preserved. Clearly the laser guiding technology gives a tremendous improvement in accelerator performance as well 
as simplifies accelerator operation and future construction (i.e.. no longer needed for transport solenofd or steering magnets). But what are the limits of the laser-guiding beam transport technique?

The strength of laser-guiding transport is initially determined by the ion linear charge density, ${ }^{3} \lambda_{i}$, where

$$
\lambda_{i}\left(\frac{\mathrm{esu}}{\mathrm{cm}}\right)=5.34 \times 10^{4} \mathrm{a}^{2} \mathrm{Pf},
$$

where $\mathrm{a}$ is the ion channel radius in $\mathrm{cm}, \mathrm{P}$ is the benzene pressure in microns and $f$ is the laser ionization fraction which is predicted by multilevel rate equations to have the dependence upon laser fluence shown in the sketch. From this information, it is apparent that modest increases in laser fluence can quickly pay off in substantial gains in ionization fraction and approach the saturated level of $\sim 20 \%$. Increased laser fluence would also allow lower operating pressure of the benzene pressure and proportionally reduce the linear growth of primary beam ionization of benzene (cross-section for electron beam ionization of $\mathrm{C}_{6} \mathrm{H}_{6}$ is $\sim 5.8 \times 10^{-18}$ ). Of greater concern is the likely exponential growth of ionization due to benzene molecular ions or fractured molecular component fons on the rematning benzene gas. Cross-sections for these processes are less well-known but the benzene ions are sloshing around in potential wells that are close to $1 M V$.

There is supportive evidence that intrabeam pulse ionization, at rates greater than linear electron beam iontzation, does occur. At the end of ATA, benzene gas limiting apertures were installed so that with suffictent pumping capacity the benzene pressure profile truncated abruptly. Laser guiding transported the beam through the accelerator but terminated at this aperture 
where the benzene pressure went to essentially zero. No magnets were used after this aperture so that the beam was allowed to freely expand into the remaining beam transport section. Current monitors measured the transmitted current as a fraction of axial distance from the aperture as the beam propagated down the $7.25 \mathrm{~cm}$ radius vacuum pipe; the results are shown in Fig. 5 . We presently have no information on the beam profile, thus time variation in the axial dependence of the fraction of transmitted current $\left(I_{B}(z>0) / I_{B}\right.$ $(z=0)$ is an incomplete data set but does suggest a time variation in beam angle, $\theta(t)$. Such $\theta(t)$ could result either from emittance increase intrapulse or intrapulse beam radius decrease due to increasing focusing resultant from ion line density increase.

Further hints of the important role of intra-pulse fonization follows from consideration of jon hose instability. For rigid electron beams and rigid ion channels, the ions sloshing in the pctential well couple to the electron beam and initiate a connective instability. The strength of coupling depends on the ion slosh time, $T_{i}$, being much shorter than the electron beam pulse duration, $T_{p} ;$ i.e.,

$$
T_{p} \gg T_{i}=\left[\begin{array}{lll}
\frac{a_{B}^{2}}{c^{2}} & \frac{m_{i}}{m_{e}} & \frac{17(k A)}{I_{B}(k A)}
\end{array}\right]^{1 / 2},
$$

where $a_{B}$ is the electron beam radius through which the fons oscillate, $m_{f}$ is the ion mass and $I_{B}$ is the bean current in kA. On ATA, experiments have been performed ${ }^{4}$ where the electron beam was perturbed and deflected from the fon column in attempts to excite ion hose. For these experiments $T_{p} / T_{q}$ has been as great as 12, yet there has been no observed ion hose growth. Analytic work backed with particle simulations ${ }^{3}$ have shown that a very powerful means of 

ejection, particularly those initiating under an accelerating gap. ${ }^{5}$ These proresses are currently under study since they seriously impact how laserguiding transport affects electron beam parameters.

\section{ADDITIONAL RESULTS: Improvements in Beam Brightness}

At ATA, progress has also been made on beam emittance and brightness. Actually, ATA's inftial concern on beam emittance did not reflect FEL goais, but rather was motivated again by beam transport issues. Before laser guiding was in place, and even prior to addressing the difficulties of the BBu instability, magnetic solenoidal transport of the beam through ATA was characterized by a continual loss of current. In Fig. 6, we show a collection of data that illustrate the problem. The experimental arrangement was that the infector, at this time equipped with a plasma surface discharge cathode, produced a beam that was accelerateo up to $6.5 \mathrm{MeV}$. At this point, a "wire zone" was located co-linear to the arelerator axis and with proper entrance and exit matching magnets. The wire zone damped out beam motion and centered the beam. ${ }^{2}$ This beam then was re-injected into the remaining part of the accelerator. As the data shows, during transport through the remaining accelerator, the centroid of the beam was confined to $\sim 1 \mathrm{~cm}$ excursions from axis, but still there was a gradual current loss. Before the wire 2one, measurements of the beam's radial proftle at various time intervals during the pulse show that there are time-varying asymmetric wings. The transport properties of the wire zone ${ }^{2}$ are such that it only damps transverse motion at the expense of increasing emittance, and that it centers the beam to axis -- it is not an emittance limiter. Particles populating these asymetric wings, after being phase mixed by the wire zone, stfil do execute sufficlently large radial excursions during 
transport to be carried to regions of poor magnetic field and eventually lost. Since small current test beams do not show anomalies in the accelerator magnetic transport or in the pulse power systems, we concluded that the far off axis particles represented characteristics of the beam as it emerged from the cathode/injector.

A collimator, even if immersed in an axial $B_{z}$ field, is a simple means of positively limiting the area in phase space populated by beam particles. ${ }^{3}$ The collimator length must be longer than the cyclotron wavelength, $\lambda_{c}$, and it is simpler to interpret, although not necessary, if the beam has sufficient energy to be emittance dominated. For a $B_{z}$-immersed collimator, the equilibrium $\left(d^{2} / d z^{2} \equiv 0\right)$ beam envelope solution is

$$
\frac{2 I_{P}}{\gamma^{2} I_{A} R_{p}}+\frac{E_{p}^{2}}{R_{D}^{3}}=1 / 9 k_{C}^{2} R_{p} \text {, }
$$

where the subscripts " $P$ " refer to pipe quantities and $I_{A}=17,000 \curlyvee$ and $k_{c}=e B / \gamma m_{0} c^{2}$. Assuming incident beam current and output collimator current are related as

$$
\frac{I_{p}}{I_{B}}=\alpha \frac{E_{p}^{2}}{E^{2}}
$$

where the weighting factor a adjust for beam distribution in phase space and $E_{D}$ refers to the phase space area (1.e., emittance) delineated by the collimator and $E$ is the full beam emittance (normalized emittance $E_{n}=Y E$ ). From this we find 


$$
I_{p}=\frac{0.727 \gamma R_{p}^{2} B_{k g}^{2}}{1+\frac{E_{n}^{2}}{2 \alpha R_{p}^{2}} \frac{I_{A}}{I_{B}}}, \begin{aligned}
& \text { if emittance } \\
& \text { dominated is } \\
& \text { proportional to }
\end{aligned} R_{p}^{4} B_{z}^{2}
$$

Because these are all "hard-edge" quantities, for a given collimator and $B_{2}$ field it is straightforward to determine, at least relatively, how much phase space is occupied by beams produced from different cathodes and injector configurations. In Fig. 7, we summarize some of the results. 6 For the collimator located $2 \mathrm{~m}$ from the injector output but always with its $\mathrm{B}_{2}$ field at $\sim 1000$ gauss, we experimented with different cathode configurations. The surface plasma discharge cathode was $25 \mathrm{~cm}$ diameter and was filled with 5000 independently power surface breakdown sites. This cathode was also operated with a control grid spaced $3 \mathrm{~cm}$ from the cathode surface. The discrete site field emission cathode was $6.7 \mathrm{~cm}$ radius and filled with carbon tufts on $0.2 \mathrm{~cm}$ spacing. Units like this with variable field emisston site spacings were tested, and as expected, the more dense the filling factor the better the beam emittance. The vacuum electric fleld stress on this cathode as well as for the uniform planar "velvet" field emission surface was $\sim 220 \mathrm{kv} / \mathrm{cm}$; both these field emission units had fleld shaping electrodes added to the cathode and anode and were operated without a control grid. Work on our development laboratory ${ }^{7}$ and supported by outside research ${ }^{7}$ indicates that after sufficient filling factor is achieved to ensure smocth E-field termination, that further increasing site concentration of emission whiskers is beneficial but only by increasing surface area for occluded gases to form, and this is what gives the beneficial increase in plasma density. As the data of Fig. 7a shows, there is an exponential like increase in beam through the collimator for these various 
cathodes. We mention that for each of these cathodes, the magnetic transport within the injector and to the collimator was adjusted for maximum current transmission with no loss. In all cases, $\sim 8 \mathrm{kA}$ was incident on the collimator and the injector was always operating at $2.5 \mathrm{MeV}$.

For completeness, in Fig. $7 \mathrm{~b}$, we show how, for a given setting of the injector magnetic transport, the ccllimator output current varies as the "Bucking Coil" current is varied. This Bucking coil is a solenold located behind the cathode and arranged to cancel the magnetic flux threading the cathode surface. This is done to zero any angular momentum contribution to the total emittance. The peak of the collimator output current versus Bucking Coll current has been verified to correspond to complete flux cancellation on the cathode surface. Finally, in Fig. 7c, we show the collimator output current dependence on the $B_{z}^{2}$ field. The departure from a $B_{z}^{2}$ fit indicates both space charge effects and that the beam does not have a uniform distribution in phase space (non-linearity in $a$ ).

SUMHARY:

Attempts to further improve the beam brightness from field emission cathodes are currently centered on the issue of how beam optics and phase mixing within the injector transport tend to "average down" the beam brightness. Particle simulation work indicates that beam brightness can be significantly improved by simply reducing the injector transport magnetic field and losing peak transport current, i.e., only transporting that high brightness portion of the total current. The simulation results shown in Fig. 8 suggest that beam brightness can be increased perhaps a factor of 5 or more simply by 
"tuning for brightness" rether than tuning for peak transported current. If this can indeed be experimentally realized and the resulting beam matched onto accelerator transport (magnetic and/or laser guided) without emittance degradation then simple field emission cathodes would, at least in the immediately near term, satisfy the needs for 10 micron FEL experiments.

\section{REFERENCES}

1. V. K. Neil, L. 5. Hall and R. K. Cooper: "Further Theoretical Studies of the Beam Break Up Instability," Particle Accelerators, 1979, Vol 9, pp 213-227.

2. 0. S. Prono, et al., "Electron Beam Guiding and Fhase Mix Damping by an Electrostatically Charge Wire," Phys. Rev. Lett., 51 (9), 723 (1983).

3. To be published work of G. J. Caporaso, LLNL.

4. To be published work of E. J. Lauer, LLNL.

5. To be published work of $r$. Rainer, J, T. Weir, Y. P. Chong, G. J. Caporaso, and A. G. Cole, LLNL.

6. Conference Paper, Q4, by 3. T. Weir; LLNL Beam Research ATA Note No. 244, Nov. 28,1984 , by 3 . T. Weir, et al.

7. Private communication with D. L. Birx, LLNL; Ph.D. thesis at MIT of David Henshelwood, released as NRI Memorandum Report 5492, "Explosive Emission Cathode Plasmas on Intense Relativistic Electron Beam Diodes," January 30,1985 .

8. Conference Paper, Y33 and Y34 by J. K. Boyd and G. J. Caporaso. 


\section{FIGURE CAPTIONS}

Fig. 1 Cutaway view of the components inside one accelerating case.

Fig. 2a Magnetic induction signals indicating growth of BBU transverse motion as the beam transports through the ATA when using customary magnetic transport.

Fig. 2b Current monttors showing pulse disruption due to BBU growth when using customary magnetic transport.

Fig. 3 The essence of laser guiding is that a laser pretonizes a small diameter plasma column and that when the relativistic beam is injected onto this column the secondary electrons are expelled having a strongly focusing, positive ion column.

Fig. 4 With laser guiding transport of the beam through the accelerator, there is minimal BBU growth and pulse disruption.

Fig. 5 Vacuum expansion of the electron beam as it exits the ion column and free transports into a six inch diameter pipe indicates that beam angles in-crease during the pulse, an observation consistent with intrapulse ionization of the benzene.

Fig. 6 Data on beam magnetic transport after a wire zone shows the centroid to be localized near axis, but still current loss down the acceleitator. The key is in the beam profiles before the wire zone which shcw significant asymetric wings (particles greatly expanding phase space) coming from the injection.

Fig. 7a For various cathode surfaces that have been tested, the current throug.l a $B_{Z}$ immersed collimator indicates the emittance and brightness.

Fig. 7b (Left) Varying the magnetic flux on the cathode surface by using a Bucking Coil changes the effective total beam emittance by canceling beam angular momentum. Figure $7 \mathrm{C}$ (Right) Varying the $B_{2}$ field over the solenoid/ collimator controls the output current in a manner that suggest non-uniform phase space distributions (nonlinear a).

Fig. B Computer simulations on how beam brightness is improved, at the cost of transported beam current, by lowering the magnetic field that is used for total peak current transport. 


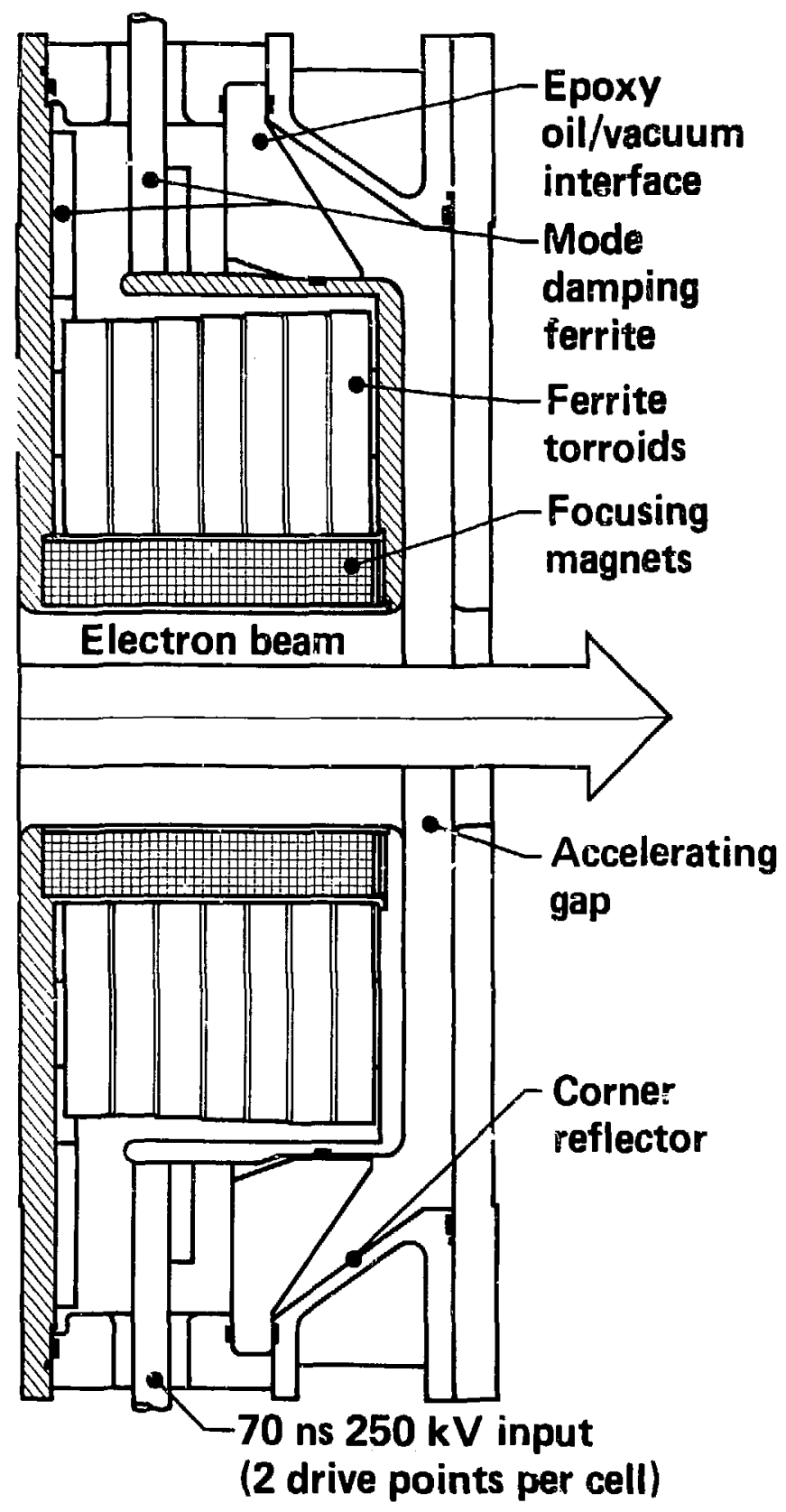

Figure 1 

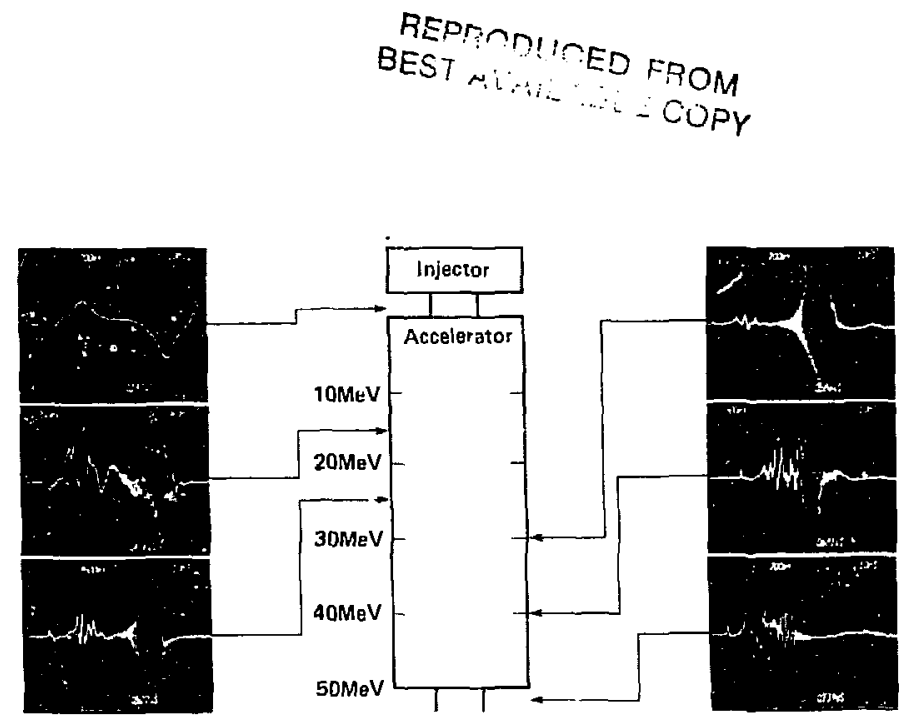

Figure 2a

- Computer plots of ATA beam current as measured by diagnostics located along the accelerator (all traces i KA/div, 10nS/div.)

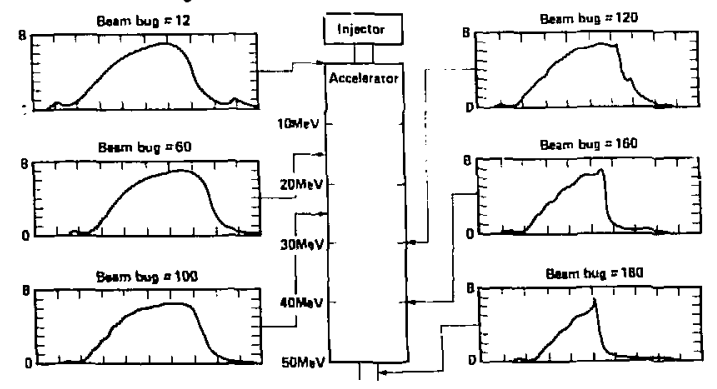

Figure 2b 


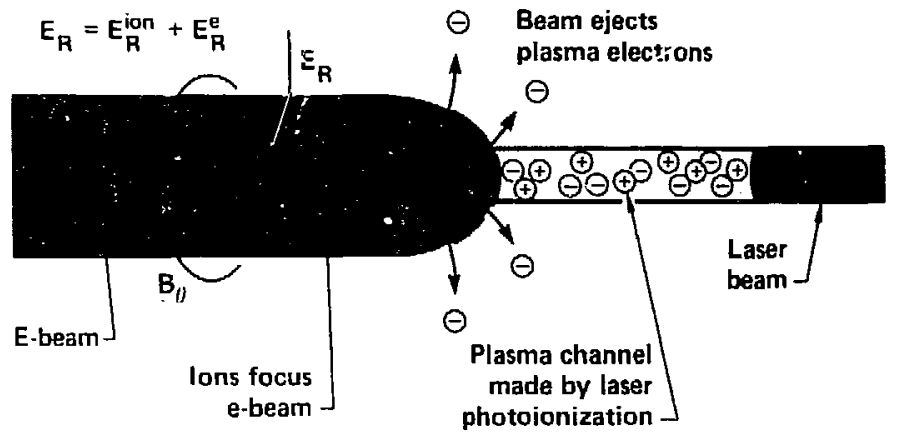

$$
\text { Net force on beam electrons } F=E_{R}^{i a n}+\underbrace{\left(E_{R}^{E}-v_{z} B_{\theta}\right)}_{\sim 0\left(1 / \gamma^{2}\right)}
$$

Figure 3

$$
\begin{aligned}
& \text { REPRODUCEO } \\
& \text { BEST AVALARE }
\end{aligned}
$$
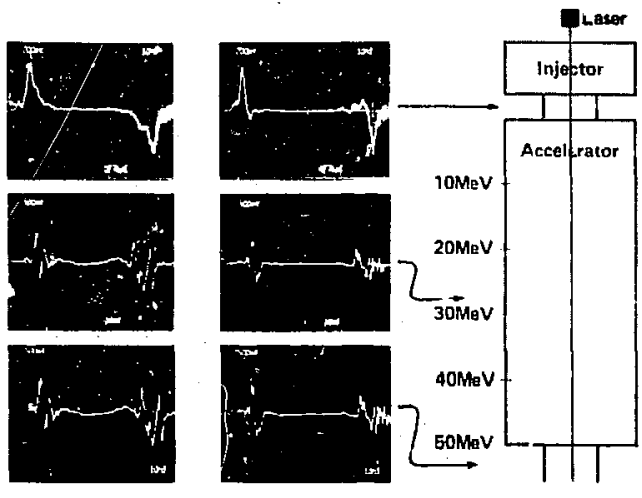

Accelerator output current $2 \mathrm{kA} / \mathrm{div}, 10 \mathrm{nS} / \mathrm{div}$.
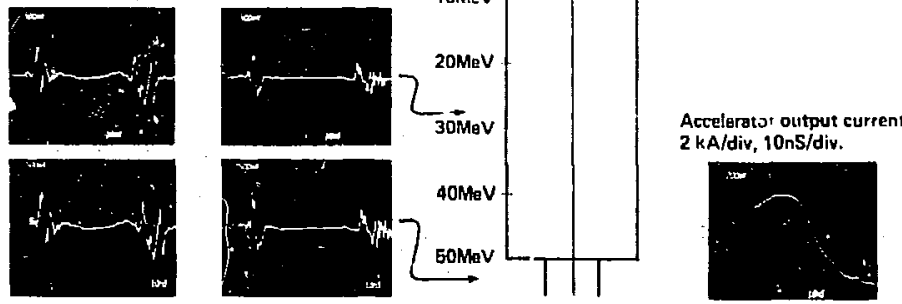

Figure 4 

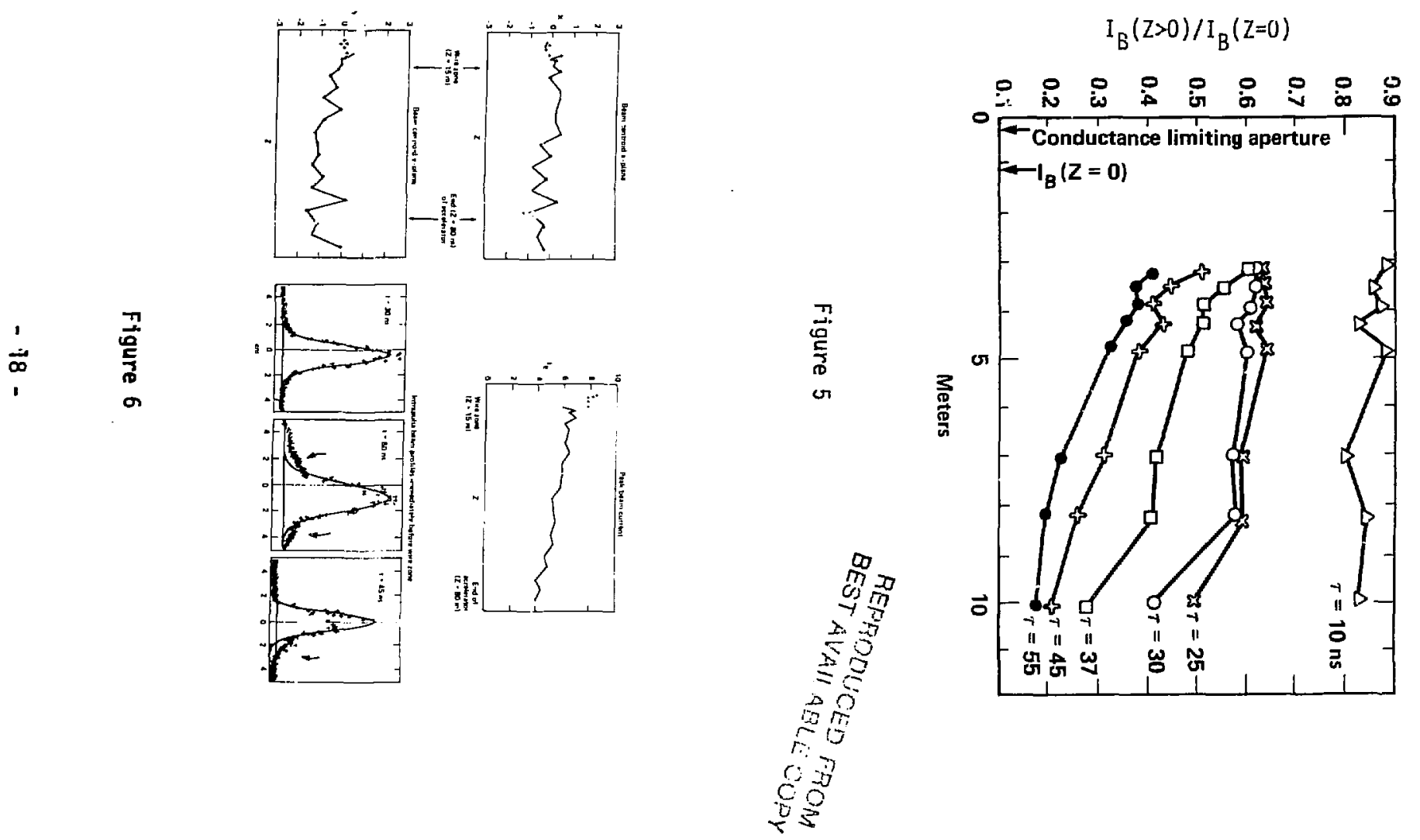


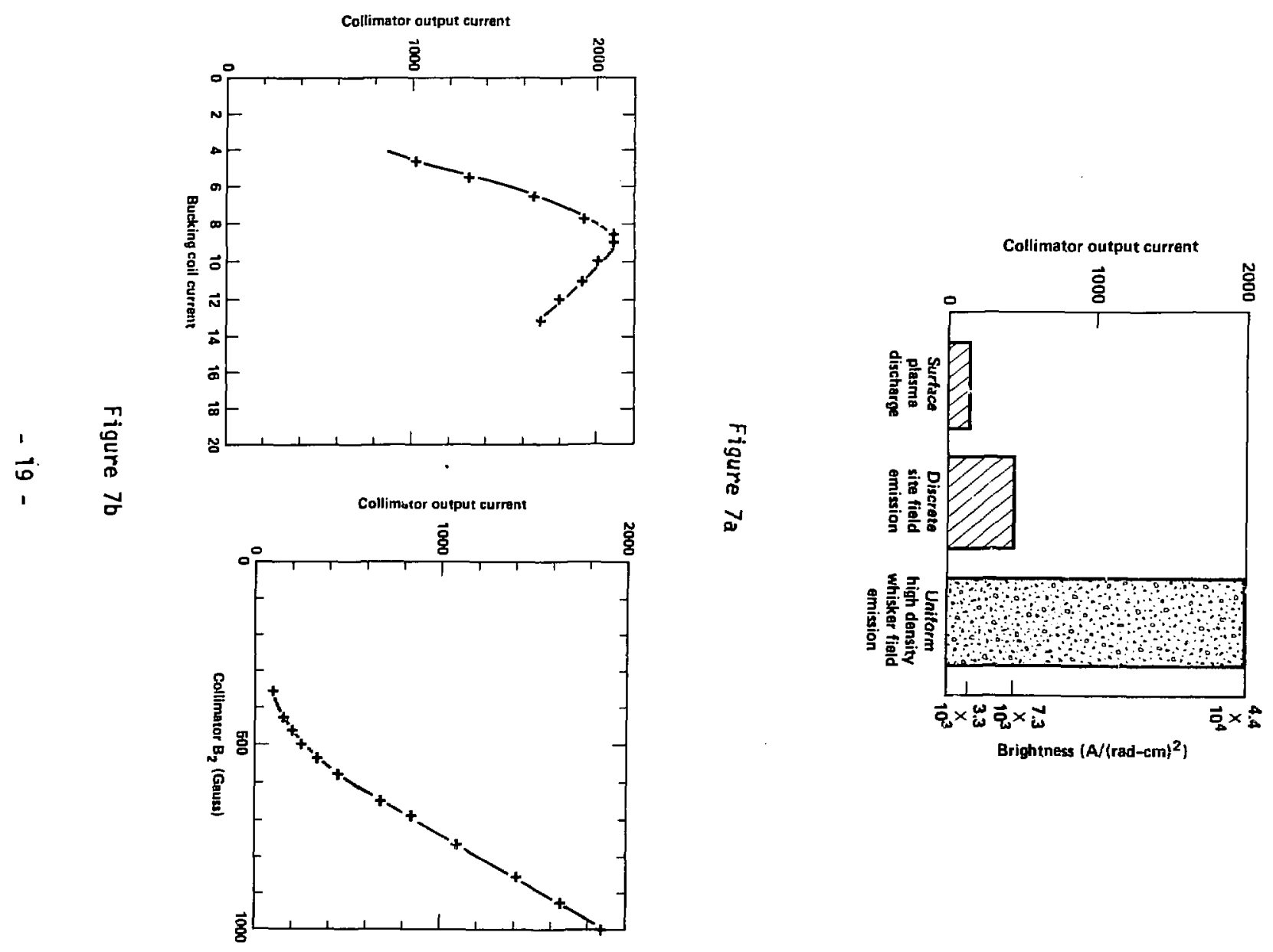




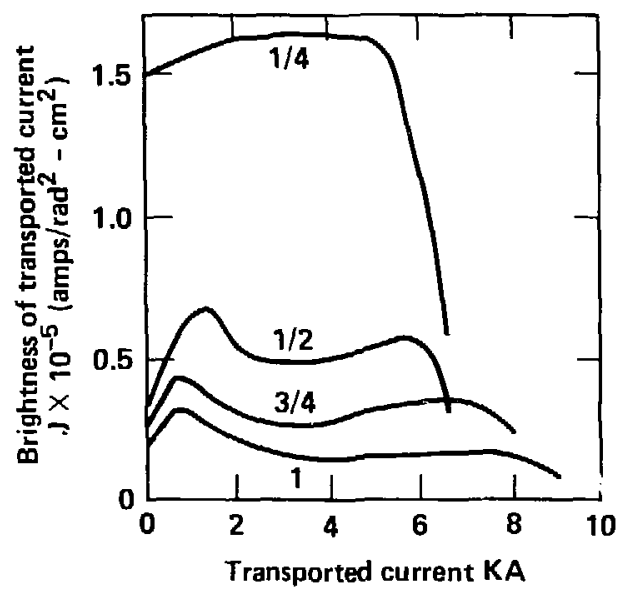

Figure 8

\section{DISCLAIMER}

This report was prepared as an account of work sponsored by an agency of the United States Government. Neither the United States Government nor any agency thereof, nor any of their employees, makes any warranty, express or implied, or assumes any legal liability or responsibility for the accuracy, completeness, or usefulness of any information, apparatus, product, or process disclosed, or represents that its use would not infringe privately owned rights. Reference herein to any specific commercial product, process, or service by trade name, trademark, manufacturer, or otherwise does not necessarily constitute or imply its endorsement, recommendation, or favoring by the United States Government or any agency thereof. The views and opinions of authors expressed herein do not necessarily state or reflect those of the United States Government or any agency thereof. 